\title{
Intelligence et artifice
}

Le Magicien d'Oz ou la simulation de l'interaction humain-machine

\section{Guillaume Alevêque}

\section{(2) OpenEdition \\ Journals}

Electronic version

URL: https://journals.openedition.org/tc/12017

DOI: $10.4000 /$ tc. 12017

ISSN: 1952-420X

Publisher

Éditions de l'EHESS

\section{Electronic reference}

Guillaume Alevêque, "Intelligence et artifice", Techniques \& Culture [Online], Varia, Online since 05 December 2019, connection on 29 September 2022. URL: http://journals.openedition.org/tc/12017 ; DOI: https://doi.org/10.4000/tc. 12017

This text was automatically generated on 29 September 2022

All rights reserved 


\title{
Intelligence et artifice
}

\author{
Le Magicien d'Oz ou la simulation de l'interaction humain-machine
}

\author{
Guillaume Alevêque
}

Objet d'un intérêt récemment renouvelé, l'intelligence artificielle (IA) est associée à un imaginaire scientifique foisonnant alimenté depuis ses débuts par des prédictions fracassantes sur l'avenir de l'Humanité et des affrontements prométhéens autour de jeux de stratégies, tels que les échecs. Les chercheurs en sciences sociales se sont largement emparés des questions soulevées par la possibilité d'automatiser la pensée (Dreyfus 1972; Searle 1980), par les impacts sociaux et économiques des développements technologiques affiliés (Casilli, 2019), ou par les représentations des chercheurs quant aux usages et applications de leurs travaux (Forsythe 2001). Mais l'IA, parce qu'elle fait référence à la création de la pensée, pose également la question d'une relation comparable à l'animisme, c'est-à-dire à «l'imputation par les humains à des non-humains d'une intériorité identique à la leur» (Descola 2005 : 229), au sein d'un domaine, la science, qui en exclut généralement la possibilité.

2 Cette question de la personnification, par ailleurs traitée dans le cas de l'anthropomorphisme des robots (Becker 2011; Grimaud et Paré 2011), nous l'approcherons ici à travers la recherche en traitement automatique du langage naturel, c'est-à-dire dans des contextes d'interactions en dialogues numériques où les éléments de communication non-verbaux sont volontairement restreints. Contrairement aux robots, en l'absence de réelle dimension iconique et de corporalité, les programmes capables de reproduire l'usage humain du langage ont un potentiel de présence (Grimaud et al. 2016 :11-17) qui ne persiste pas hors de leur utilisation. Un ordinateur éteint ne présage pas de l'existence en son sein d'agents conversationnels. Néanmoins, parce qu'ils ont la faculté de communiquer par le langage, ceux-ci se voient très aisément attribués le statut de personne, mais également la faculté de raisonner.

3 Grâce à cette spécificité d'une présence qui ne se concrétise qu'à travers son dialogue avec ses interlocuteurs, s'est développée une forme d'expérience qui a discrètement accompagné la recherche en IA depuis les années 1970: le Magicien d'Oz. Cette expression désigne un dispositif où des volontaires sont invités à tester les fonctionnalités d'un outil informatique. Cependant, l'outil informatique en question 
n'existe pas et c'est en réalité un être humain - le magicien - qui en endosse le rôle. Autrement dit, il s'agit d'une expérience qui étudie l'interaction entre l'humain et la machine sans machine, mais à laquelle est malgré tout attribuée une portée heuristique.

L'hypothèse présentée ici est qu'en proposant une interaction entre humain et nonhumain inédite au sein de la science, le Magicien d'Oz révèle des logiques de projection et d'imitation qui plus généralement, semblent faire la spécificité de la recherche en IA. D'une part, le Magicien d'Oz apparaît comme un mode d'expérience qui en circonscrit la logique première, car il est uniquement valide lorsqu'il est envisagé qu'un être humain puisse imiter les fonctionnalités d'une machine qui, dans le futur, imitera les siennes. D'autre part, la relation entre imaginaire scientifique et innovation technique prend une forme sans précédent ${ }^{1}$, au sens où l'expérimentateur assume le rôle d'une machine qui n'existe pas encore et teste ainsi la validité d'une innovation dont la faisabilité technique n'est pas encore assurée. En jouant le rôle de la machine, l'être humain devient un temps l'indice d'un prototype ${ }^{2}$ - l'IA - dont il est habituellement le modèle.

5 Après avoir situé le Magicien d'Oz dans le contexte historique de la recherche en IA, nous décrirons ses champs d'application en étudiant différents projets qui ont conduit cette expérience. Enfin, l'analyse portera sur la logique de «jeu» (Bateson 1977) impliquée dans cette pratique scientifique en l'envisageant comme une forme de ritualisation articulée autour d'une « personne-objet », en reprenant le concept d'objetpersonne de Carlo Severi (2017).

\section{Contexte}

6 Certains auteurs font remonter l'origine de la recherche en IA à Aristote et à ses syllogismes. La volonté de produire des raisonnements logiques universaux représenterait les prémisses de la possibilité d'automatiser le raisonnement et de le matérialiser en programmation informatique sous la forme propositionnelle «si... alors... » (Marquis, Papini et Prades 2014 : 2 ; Russel et Norvig 2010 : 6). D'autres auteurs sont fréquemment cités, en particulier Thomas Hobbes qui conçoit le raisonnement comme une forme de calcul et associe ainsi les processus de la pensée à la computation. Mais c'est au milieu du $\mathrm{XX}^{\mathrm{e}}$ siècle que ce domaine de recherche connaît un essor fondateur avec l'émergence de la cybernétique, de la théorie de l'information et des travaux d'Alan Turing. Ce dernier posa les bases de la recherche en IA, dès les années 1940, en étudiant la notion de calculabilité, en concevant sa «machine» (qui n'est pas une machine, mais un objet mathématique abstrait), en formalisant la notion d'algorithme et enfin en proposant son jeu de l'imitation, plus connu sous le nom de test de Turing (Lassègue 1998).

7 L'expression «Intelligence Artificielle » et sa définition comme domaine de recherche ont pour leur part été forgées en 1956 au Dartmouth College dans le cadre d'un séminaire pluridisciplinaire dont l'objectif a été résumé ainsi :

L'étude reposera sur la conjoncture que chaque aspect de l'apprentissage ou de toute autre facette de l'intelligence peut être décrit en principe si précisément qu'une machine puisse être construite pour le simuler. On tentera de proposer des solutions pour que les machines puissent utiliser le langage, former des abstractions et des concepts, résoudre des types de problèmes réservés aux humains pour l'instant et se perfectionner. (cité par Russell et Norvig $2010: 18$ ) 
Dans ce vaste ensemble d'intérêts et de prospectives théoriques et appliquées, c'est la question du langage qui nous intéresse particulièrement. Dès les années 1950, le lien entre langage et pensée est identifié comme un enjeu crucial, mais en pratique la question demeure occultée par d'autres nécessités relevant de la stabilité des systèmes logiques. Surtout, au sein de la recherche fondamentale, créer un programme et communiquer avec lui ont longtemps été en équivalence à travers la formalisation et l'application des langages informatiques. Intégrer la question du traitement automatique du langage naturel impliquait donc de concevoir des programmes non seulement aptes à répondre à des commandes, mais également à même de traiter l'ensemble des liens entre lettres, mots et phrases. La technique du Magicien d'Oz, c'est-à-dire le dispositif scientifique reposant sur la substitution d'un être humain à outil informatique, apparait au moment où se croisent les enjeux de ces deux formes de communication avec la machine (langage de programmation et traitement du langage naturel).

\section{Le Magicien d'Oz}

9 Le document le plus ancien faisant référence à ce type de pratiques est un rapport interne du centre de recherche Thomas. Watson d'IBM datant de 1976. La problématique des auteurs, Ashok Malhotra et Peter Sheridan (1976), est de déterminer le cahier des charges d'un programme avant de pouvoir en tester les qualités de fonctionnement. Pour cela, ils proposent une "méthodologie» en connectant deux terminaux grâce auxquels un « sujet » interagit avec un « expérimentateur » qu'il croit être un programme informatique. Les échanges qui en résultent doivent ainsi permettre d'identifier les caractéristiques des commandes requises préalablement à la programmation du logiciel.

10 Dans le même temps, le groupe de recherche en ergonomie d'Alphonse Chapanis à Hopkins développe une technique similaire nommée "experimenter in the loop technique » (Green et Wei-Haas 1985). C'est au sein de ce groupe que serait apparue la référence au Magicien d'Oz (Baum, 1900), dans le cadre de la thèse de Jeff Kelley au début des années 1980. Lors d'un séminaire, celui-ci aurait comparé la situation d'un expérimentateur démasqué à la principale révélation de l'intrigue du Magicien d'Oz où les pouvoirs du puissant magicien se révèlent être des illusions élaborées par un simple être humain. Dans le livre, la prestidigitation tient de la mise en scène (il s'agit de décor, de mannequins et de ventriloquie). Dans le film de 1939 qui popularisa cette œuvre au Etats-Unis, le magicien produit ses illusions grâce à une machine. L'expression et la technique qui s'y réfère furent ainsi popularisées par Jeff Kelley et Alphonse Chapanis lors de colloques, même si d'autres chercheurs conduisirent des expérimentations fondées sur ce même principe au début des années 1980 sans faire référence à ce nom, en particulier John D. Gould et al. (1983) et Elizabeth Zoltan-Ford (1984).

11 Cette référence à l'intrigue centrale du Magicien d'Oz n'est pas la seule à émerger à cette période en informatique. Il y a également le néologisme PNAMBIC (pour «Pay no attention to the man behind the curtain ", réplique célèbre du magicien au moment où son stratagème est découvert par Dorothy). Elle fait néanmoins référence à un ensemble de pratiques qui dépasse le cadre de l'expérience. En effet, Daniel Klein (1981 :112) a forgé le terme pour qualifier un stage de conception où un système présenté 
comme complètement automatique requiert en réalité une intervention humaine pour accomplir le résultat désiré.

Quoiqu'il en soit, l'expression «Magicien d'Oz» a conceptualisé une pratique jusqu'alors peu explicitée. En cela, elle contribua à sa formalisation et sa légitimation comme un protocole expérimental scientifique permettant de faire avancer la connaissance et d'améliorer la conception des programmes.

\section{Principes et applications}

13 À l'origine, le Magicien d'Oz relève d'une logique d'ingénierie. Il s'agit d'anticiper les fonctionnalités d'un programme, d'améliorer son ergonomie et la pertinence de son champ d'application et ceci à moindre coût. La mémoire des ordinateurs étant alors extrêmement limitée et il nécessaire de déterminer les requêtes et les mots les plus pertinents à intégrer au programme préalablement à sa conception.

14 Jeff Kelley par exemple utilise le Magicien d'Oz pour déterminer les usages et les pratiques que ses " sujets " associent à l'agenda électronique qu'il est en train de développer. Au fur et à mesure de la conception du programme, les fonctionnalités devant être simulées par le magicien se réduisent, jusqu'à ce que son intervention ne soit plus nécessaire.

Progressivement cependant, la pertinence scientifique de cette technique a été considérablement élargie. En effet, l'innovation constante de l'informatique a rapidement confronté les chercheurs à la question de savoir " comment étudier l'activité d'opérateurs dans une situation qui n'existe pas encore » (Salembier 191: 552). Dans les années 1980, «l'utilisabilité » (Chapanis 1984) des ordinateurs s'impose comme une préoccupation majeure de l'ergonomie et de la recherche appliquée en interaction Homme-Machine (H-M) relativement aux nouveaux débouchés commerciaux de l'informatique que représentent les entreprises. Dans ce contexte le Magicien d'Oz répond à une problématique prédictive, en proposant d'anticiper par simulation la communication entre les humains et les futures machines. Cet objectif scientifique a rapidement pris le pas sur l'usage du Magicien d'Oz comme aide à la programmation informatique. Cela ne signifie pas que cet aspect ait disparu des procédés de conception expérimentale, mais que nos sources - les communications scientifiques - ne le mentionnent plus, car il a perdu son caractère innovant . A l'inverse, il est toujours d'actualité en tant qu'expérience et quelques exemples vont nous permettre de mieux cerner son application.

\section{«Une machine à écrire à l'écoute » (1983)}

16 Les travaux de John D. Gould au sein d'IBM, avec John Conti, et Todd Hovanyecz au début des années 1980, portent sur le développement d'un terminal de traitement de texte à commande vocale (Gould et al. 1983). L'outil que représente la machine à écrire n'a pas encore été tout à fait intégré à l'ordinateur et certains modèles électroniques sont encore en développement. Ils envisagent la "machine à écrire à l'écoute " (Listening tapewriter: ill.1) comme un outil révolutionnaire qui combinerait les intérêts de l'oral et de l'écrit, accélèrerait la communication et faciliterait le travail de l'auteur tout en réduisant les couts (ibid. : 295). John Gould et ses collègues (1983) concèdent que 
l'état de la reconnaissance automatique de la parole n'est pas assez avancé pour concevoir une telle machine. Ils estiment néanmoins que la simulation d'une interface utilisateur pourrait permettre de guider et d'influer le développement de la technologie lorsque celle-ci sera disponible.

L'étude compare ainsi les performances et les retours d'expériences auprès de cadres d'entreprise vis-à-vis de cette machine à écrire à l'écoute par rapport aux méthodes traditionnelles de composition (écriture ou dictée). Le participant est invité à dicter un courrier dans un microphone. Le logiciel de reconnaissance vocale est joué par une dactylographe qui, cachée dans une pièce adjacente, tape le courrier dans un terminal relié à l'écran du participant. Différents scénarii de logiciels sont comparés sur ce principe, le magicien (la dactylographe) simulant des erreurs (d'homonymie...) illustrant les degrés de perfectionnement testés. Les auteurs concluent que l'avis des utilisateurs sur les performances du système par rapport aux méthodes existantes dépend des contraintes imposées simulant une machine plus haut moins avancée, autrement dit, plus ou moins proche des compétences réelles de la dactylographe ellemême.

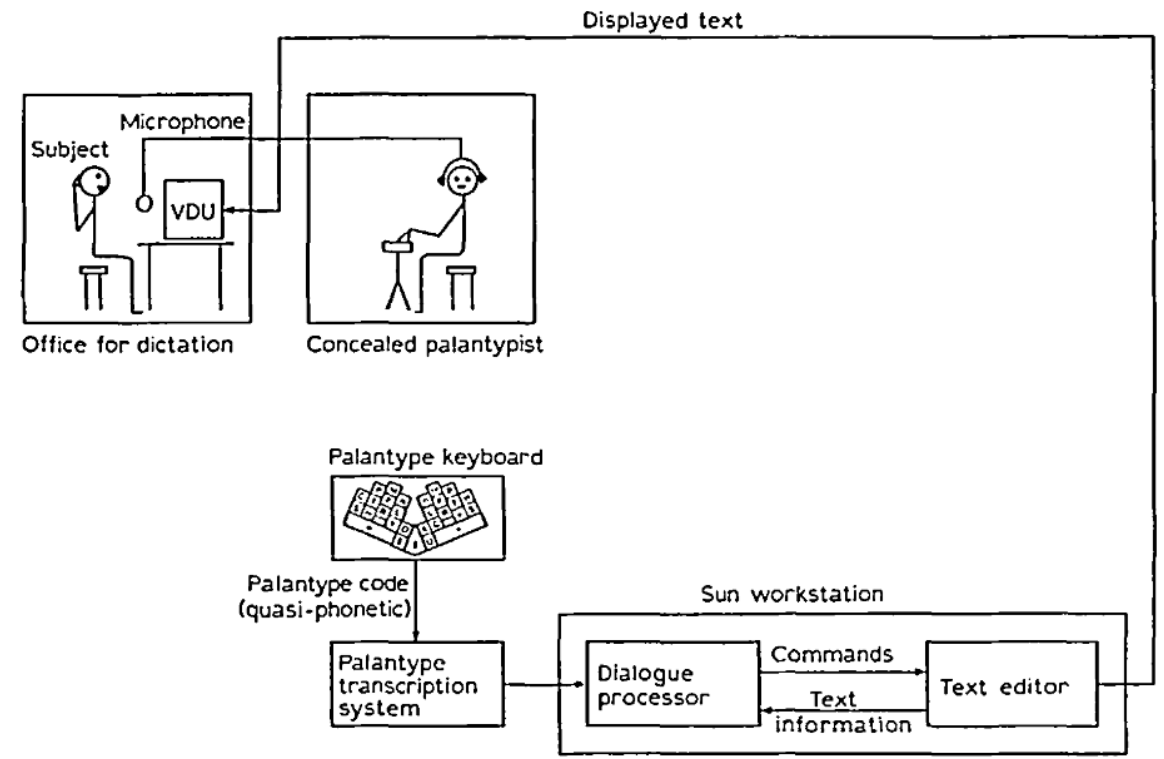

III. 1 : Schéma d'une simulation de machine à écrire à l'écoute (Newell et al. 1990 : 10).

\section{Un logiciel d'aide aux voyageurs (1993)}

Dix ans plus tard, Nils Dahlbäck, Arne Jönsson et Lars Ahrenberg de l'université Linköping en Suède publient un article (1993) qui, tout en se basant sur une étude de cas - un programme d'aide pour voyageur - interroge essentiellement la méthode du Magicien d'Oz, sa mise en œuvre et son intérêt. Par rapport aux travaux de John Gould et de ses collaborateurs (1983), le périmètre heuristique de l'expérience se déplace sensiblement. Déjà, ces derniers ne simulaient plus un programme comme une étape de test d'un projet de logiciel. Son anticipation avait acquis un degré d'abstraction, puisque l'expérience devait établir l'intérêt de la conception future d'une telle machine, dont il avait l'image mentale (le prototype au sens de Gell 2009), mais qu'il 
n'était pas en mesure de concrétiser (d'en produire un indice) autrement que par un subterfuge.

Dans l'expérience de Nils Dahlbäck et al. (1993), la machine n'est plus le prolongement d'une action humaine telle que recopier un texte dicté. Il s'agit d'un système ayant une expertise, en l'occurrence des informations sur des destinations de voyage. Surtout, le développement de logiciel est en réalité un prétexte pour étudier l'interaction $\mathrm{H}-\mathrm{M}$ et la pertinence de sa simulation:

Il a souvent été avancé [...] qu'un dialogue naturel entre un humain et une machine devrait ressembler autant que possible à un dialogue entre êtres humains. Cependant [...] un ordinateur n'est pas une personne, et certaines différences sont telles que l'on peut s'attendre à ce qu'elles aient une influence majeure sur le dialogue. Par conséquent, produire des données à partir d'interactions humaines n'est pas une source fiable d'information pour certains aspects importants de la conception, et en particulier du style et de la complexité de l'interaction. (ibid. : 258)

Par conséquent, pour les auteurs, tout porte à croire que des ajustements dans notre comportement linguistique seront accomplis durant les interactions H-M. La technique du Magicien d'Oz serait alors la meilleure alternative disponible pour découvrir par anticipation les caractéristiques linguistiques spécifiques à ce type d'interaction du point de vue de l'humain ${ }^{3}$.

21 Néanmoins les auteurs avouent leur impossibilité à analyser les données qu'ils ont obtenues lors de leur test ( 150 dialogues en tout) en l'absence de théorie à tester concernant le dialogue multimodal. Ils réaffirment pourtant la pertinence de leur démarche. Selon eux, il est en effet nécessaire de simuler des systèmes futurs plutôt que d'étudier l'interaction H-M par le biais des systèmes existants, car les humains s'adaptent aux programmes et à leurs limitations. La simulation s'impose donc pour déterminer ce dont ils auraient idéalement besoin. Ceci exprime une certaine utopie du progrès technologique. Les auteurs s'apparentent à des précurseurs ayant la volonté de faire abstraction des contraintes techniques du moment pour anticiper la meilleure voie vers laquelle l'innovation devrait tendre. La "machine» est un artefact à venir, dont on ne mesure pas encore les spécificités en termes d'interactions, mais qui devrait être orientée au mieux par rapport aux futurs besoins des utilisateurs. Nous y reviendrons, mais il s'agit là d'un cas particulier où l'objet est simultanément appréhendé comme un agent et un outil.

Quoiqu'il en soit, Nils Dahlbäck et ses collaborateurs (Dahlbäck et al. 1993) offrent également de précieux conseils méthodologiques aux futurs magiciens. En effet, la conception de ce type d'expérimentation implique aussi bien la détermination du cadre de l'expérience que certains préparatifs de mise en scène, afin de produire des données valables. Ainsi, pour limiter le risque de tirer des conclusions généralistes qui ne seraient que le reflet de protocoles expérimentales spécifiques, les auteurs préconisent de varier les versions du programme, autant dans son contenu que dans son "intelligence", son vocabulaire et les actions disponibles. De surcroit, la tâche proposée par le scénario ne devrait être ni trop facile ni trop dure, afin que les données puissent être collectées en quantité suffisante. Sans négliger que des instructions trop détaillées induiraient le risque que l'utilisateur se réapproprie les expressions employées par les chercheurs biaisant ainsi l'interaction H-M en laboratoire. Ils précisent enfin que l'utilisateur ne devrait pas être familiarisé avec le programme mais 
avoir une connaissance du domaine d'application afin que les réponses ne soient pas contrôlées consciemment et volontairement (ibid. : 265).

Concernant la mise en scène, les auteurs relèvent un point particulièrement sensible : comment joue-t-on le rôle d'une machine? L'expérience reposant sur le fait de ne pas être démasqué, des accessoires s'imposent. Ainsi, pour que l'humain ressemble à la machine (qui imite l'homme), un logiciel d'aide spécifique est nécessaire avec des éléments de réponses préenregistrés et ordonnés en suggestions. Ce programme permettrait par exemple d'inscrire un message «Wait " pour indiquer que la requête est prise en compte ou de simuler un bug et un redémarrage du système si le magicien ne sait pas y répondre.

Enfin, les auteurs reconnaissent qu'il est très difficile pour un magicien de «maintenir une performance cohérente » afin de ne pas être démasqué, mais également pour que la simulation soit valide. Il s'agit par exemple de donner la même réponse de la même manière à un même utilisateur tout autant qu'à des utilisateurs différents afin de pouvoir généraliser les résultats obtenus à partir des différents dialogues.

\section{Un service de renseignement par téléphone (1997)}

La nécessité d'expérimenter plutôt que de se baser sur des intuitions et des jugements pour concevoir des systèmes informatiques en langage naturel est partagée par Norman M. Fraser et Nigel Gilbert (1991) qui considèrent que cela tend à créer des systèmes incomplets reflétant les engouements et les désintérêts thématiques des chercheurs. Selon eux, pour connaître les caractéristiques du dialogue H-M avant la conception des programmes, il est nécessaire de les simuler. C'est ce qu'ils entreprirent par la suite en collaboration avec Robin Wooffitt et Scott McGlashan dans le cadre du projet SUNDIAL. L'ouvrage qui en résulta, Human, computers and wizards. Analysing human (simulated) computer interaction (Wooffitt et al. 1997) est, à ma connaissance, le seul dont le Magicien d'Oz est le thème principal. Leur analyse se fonde sur une critique de l'approche cognitive de l'interaction H-M et en particulier de la méthode GOMS (Card, Moran et Newell 1983) utilisée pour gagner en productivité bureautique. Robin Wooffitt et al. (1997) considèrent qu'en se focalisant sur l'aspect psychologique de l'interaction, celle-ci est extraite de son contexte sociologique. Pour le mettre en évidence, ils font le choix de traiter l'interaction $\mathrm{H}-\mathrm{M}$ à partir d'une analyse conversationnelle.

26 A partir de Harvey Sacks (1992) et Emanuel Schegloff (1968), ils s'intéressent à la structure de la conversation et notamment aux paires adjacentes (salutationsalutation ; requête-acceptation/rejet ; question-réponse). Pour cela ils optent pour une étude comparative entre des dialogues enregistrés par téléphone avec le service de renseignements téléphoniques de British Airways et le même service simulé en laboratoire pris en charge par une machine.

Parmi les éléments d'interaction relevés qui ne semblent pas liés à la performance du magicien (lenteur des réponses...), il faut noter que lorsque le participant considère que sa requête n'est pas appropriée au service de renseignement simulé, il en présente le caractère problématique implicitement à un être humain, mais explicitement à une machine (Wooffitt, $1997: 88$-89). On peut considérer que c'est un élément central de 
différenciation : on pense pouvoir convaincre ou s'arranger avec quelqu'un, pas avec une machine.

Quoiqu'il en soit, une des observations principales mises en avant par les auteurs est la persistance des éléments de politesse du dialogue humain alors que les sujets pourraient aussi bien dire « bonjour » et « merci » à une bouilloire (ibid. : 167). Il semble cependant nécessaire de souligner ici que ces auteurs occultent un élément important de l'interaction qu'ils simulent. En effet, les dialogues qu'ils enregistrent et analysent prennent place dans un contexte particulier et en présence d'autres acteurs. En effet, l'expérience a lieu sur un campus universitaire où les volontaires - des étudiants - ne font pas seulement face à ce qu'ils pensent être une machine, mais tiennent également compte dans leurs interactions, des chercheurs qui les encadrent. Ceux-ci peuvent bien être absents ou derrière une vitre sans teint, l'expérience est un cadre d'action dont ils sont en finalité les destinataires. Ainsi, par le biais de la simulation, se rejoignent un cadre expérimental où les principaux agents s'effacent et un subterfuge qui fait intervenir une machine factice. De nos jours, ce dispositif est encore utilisé, bien que le perfectionnement des programmes d'IA éloigne de plus de plus la performance des magiciens du rôle de robot.

\section{La personnalité des chatbots (2017)}

Dans l'optique proposée par Robin Wooffitt et ses collègues (Wooffitt et al. 1997), les travaux plus récents étudient les ordinateurs comme des « acteurs sociaux » (Reeves \& Nass 1996), en considérant que les utilisateurs tendent à appliquer inconsciemment certaines règles sociales aux technologies et les appréhendent comme des êtres vivants. Cependant cela dépend également de la capacité du programme à être poli et coopératif, à avoir de l'humour et de l'empathie (Klein et al. 2002). Cela dépend également de l'image qui lui est conférée (Corti et Gillespie 2016). Plus généralement, une personne construit une représentation de son interlocuteur machine à travers les compétences linguistiques et techniques dont elles témoignent durant l'interaction (Amalberti et al. 1993).

Dans ce contexte, une branche de l'IA s'est progressivement développée autour de l'imitation de la conversation humaine en programmant des agents conversationnels ou chatbots. Parmi les ancêtres notables des chatbots, il faut mentionner ELIZA et ALICE (Weizenbaum 1966 ; Wallace 2008 :181-210) qui introduisirent dans l'interaction $\mathrm{H}-\mathrm{M}$ des stratégies de réponses pour masquer leurs difficultés conversationnelles (répéter, s'indigner, éluder...). Ces dernières années, des innovations importantes ont eu lieu grâce à la quantité phénoménale de données disponibles en langage naturel sur internet. Celles-ci ont permis d'entrainer des chatbots basés sur des algorithmes devant apprendre de leurs propres conversations avec les utilisateurs de réseaux sociaux (de type Twitter). Le programme le plus connu à ce titre est celui de Microsoft dont la version en mandarin, Xiaoice, revendique des dizaines de millions d'utilisateurs ${ }^{4}$, alors que la version anglaise Tay a très rapidement été " rebootée » après avoir formulé des messages racistes et sexistes (Neff \& Nagy, 2016). Notons que Xiaoce et Tay ont appris à converser (à imiter la conversation) dans des contextes opposés quant à l'anonymat et la liberté d'expression. On peut ainsi considérer que ces chatbots ne sont pas tant le reflet d'êtres humains que des spécificités de l'internet au sein duquel ces humains interagissent. C'est en ce sens qu'ils sont socialisés. 
31 De telles avancées technologiques pourraient faire accroire que la simulation de l'interaction H-M n'a plus de pertinence étant donné le degré de sophistication des agents conversationnels et leur capacité à dialoguer simultanément avec un grand nombre d'utilisateurs ${ }^{5}$. Le Magicien d'Oz a pourtant fait l'objet de communications récentes en relation avec les chatbots. Ainsi, Indrani Medhi Thies, Nandita Menon, Sneha Magapu, Manisha Subramony, et Jacki O’Neill ont conduit une expérience au centre de recherche de Microsoft à Bangalore portant sur la personnalité des Chatbot (Medhi Thies et al. 2017). Le propos semble sensiblement être le même : les systèmes d'IA devenant de plus en plus performants, l'enjeu est de comprendre l'objectif que devraient avoir les concepteurs même si celui-ci n'est pas encore réalisable (ibid. : 445). Plus précisément, il s'agit de déterminer la personnalité d'un agent conversationnel qui aurait la faveur des jeunes indiens urbains. Pour cela les auteurs comparent trois versions: Maya, un bot de recommandation astucieux («productivity bot with nerd wit») qui plaisante, fait attention aux intérêts de l'utilisateur et propose des liens vers des pages internet dès que la conversation s'y prête. Ada, drôle et charmeuse ("funflirtatious »), utilise beaucoup d'émoticônes. Et Evi, bonne copine sensible (« emotionnal buddy bot ») et empathique, essaie de donner le sentiment qu'elle est toujours à l'écoute en disant souvent « always there for you ».

32 Ces éléments de conception qui définissent les "personnalités » sont autant d'indications pour les rôles endossés par le magicien d'Oz (la magicienne en l'occurrence). Il ne s'agit plus de simuler un robot impersonnel (comme chez Wooffitt et al. 1997), mais d'interpréter des personnalités artificielles.

En termes de résultats, les utilisateurs ont indiqué préférer un chatbot qui apporte de la « valeur ajoutée à leur vie » en proposant des recommandations en accord avec leurs centres d'intérêts (Maya). Avec le temps, ils pensent pouvoir s'ouvrir sur des sujets plus personnels (Evi) dont ils ne discuteraient pas avec leurs amis humains (Medhi Thies et al. 2017: 457). Autrement dit, il s'agirait d'un agent complexe regardé par les utilisateurs comme suffisamment hors du social pour leur permettre de se confier tout en n'ayant pas peur de perdre la face (au sens de Goffman 1974).

À l'instar des travaux de Robin Wooffitt et al. (1997), cette expérience renvoie à la problématique du contexte. Les auteurs n'ont pas pris en compte leur propre présence dans le processus d'enquête. En effet, l'expérience est accomplie dans un cadre qui dépasse la seule interaction entre le volontaire et la machine simulée. Elle s'établit dans un contexte où il n'est peut-être pas socialement aisé de dire à des chercheurs que l'on a préféré le chatbot aguicheur. Autrement dit, un contexte social où l'utilisateur pourrait bien être en mesure de perdre la face.

Que retenir de ce panorama de la recherche en interaction $\mathrm{H}-\mathrm{M}$ simulée ? Relativement à la pratique de la science, le Magicien d'Oz est à la croisée de différentes logiques : celle de la simulation, du prototypage et de l'expérience. En tant qu'expérience, la technique se veut extraite du contexte social de son exécution. Mais l'interaction à la machine au cours d'une expérience de laboratoire est difficilement comparable à ce qu'elle peut être dans l'intimité ou l'anonymat. Cela est valable qu'il s'agisse de machine réelle ou simulée. Néanmoins, avec la logique de prototypage du Magicien d'Oz, la matérialisation du futur doit passer par le biais d'un être humain imitant une machine censée l'imiter. Cela situe la simulation dans un contexte très particulier parce qu'il est impossible de simuler une interaction sociale sans que celle-ci s'en trouve de 
fait enchâssée dans d'autres. Ce type de contexte renvoie à la théorie du «jeu » de Gregory Bateson et aux développements relatifs à la ritualisation qui s'y réfèrent.

\section{Simuler la machine : jeu et ritualisation}

Le « jeu», au sens de Gregory Bateson, n'est pas une activité, mais une modalité de communication par l'action. Pour Gregory Bateson, certaines interactions sont porteuses d'un méta-message ${ }^{6}$ indiquant " ceci est un jeu ", c'est-à-dire : "les actions auxquelles nous nous livrons maintenant ne désignent pas la même chose que désigneraient les actions dont elles sont des valant pour " (Bateson $1977: 211$ ). En ce sens, le jeu, en tant que cadre fictionnel, est par essence ambigu: il manifeste simultanément une filiation et une distanciation par rapport à l'action à laquelle il se réfère; il indique à la fois une assimilation et une distinction (Bateson 1977 : 216). À la suite de Gregory Bateson, Erving Goffman désigne comme modalisation cette transformation par laquelle une action en devient une autre, tout en lui restant associée (1991: 52).

Bien que cela soit rare, le jeu est une catégorie d'action qui peut être présente dans les dimensions techniques et expérimentales de l'activité scientifique. C'est particulièrement en le cas en IA et en robotique où le principe d'imitation est au cœur des démarches de conception, mais également en termes d'application et de communication. En IA, l'affrontement aux échecs ou dans le cadre du test de Turing s'est imposé comme le moyen de juger des progrès de la recherche. En robotique, comme le souligne Joffrey Becker (2011), la mise en scène et en acte des robots enchâssent du jeu dans du jeu dans une relation à trois termes, entre ingénieurs, robots et public. Dans le cas des chatbots, on retrouve cette même relation entre imitation et efficacité technique et sociale. Son champ d'application est néanmoins différent. Il s'agit d'imiter au mieux une compétence, le langage, dans son usage social, la conversation. Le principe minimal est de parvenir à occulter le fait que la reconnaissance des signifiants ne présume pas de la compréhension des signifiés, même si l'être humain a tendance à l'inférer au cours de l'interaction.

Le premier chatbot reconnu et qui a aussi bien servi d'inspiration que de code source à ses successeurs est ELIZA (Weizenbaum 1966). Il a été conçu dans les années 1960 sur la base de la reconnaissance de mots clés dans des phrases dactylographiées, auxquels étaient associées des règles de réponses. Les contraintes techniques d'ELIZA - une base de données de cinquante de mots-clés, dont l'identification déclenche une reformulation selon un nombre de scripts limités - ne l'a pas empêché de rencontrer un véritable succès, a-t-elle point que Joseph Weizenbaum s'est investi par la suite à tenter de démystifier sa machine et l'IA en général. En effet, ce programme, surnommé DOCTOR, éveilla aussi bien l'intérêt de psychiatres qui y voyaient un futur assistant permettant de traiter plus rapidement un nombre accru de patients, que des employés du MIT qui s'y confiaient en cachette, ce que Joseph Weizenbaum découvrit lorsque des membres du personnel s'opposèrent à l'extraction de l'historique des conversations de son programme (ibid.).

L'astuce principale d'ELIZA réside moins dans son code, que dans l'adéquation de celuici au cadre attribué à l'interaction. Au préalable, en effet, l'interlocuteur est invité à converser avec ELIZA comme s'il s'agissait d'un psychiatre. A travers ce contexte, c'est la socialisation de la machine qui donne l'illusion que celle-ci puisse comprendre la 
signification du dialogue. Il s'agit au départ d'un truc (Grimaud : 2006) à partir duquel Joseph Weizembaum espère occulter au mieux les faiblesses de son programme, dans un cadre où faire parler la personne de soi permet de focaliser le nombre de mots-clés sur des thèmes structurants (travail, famille...) et où la reformulation est un mode de conversation approprié. Mais celui-ci ne dévoile toute l'étendue de son efficacité qu'à l'usage, car cette reformulation est socialement valorisée et liée à un savoir curatif fondé sur la possibilité d'interpréter les réponses du médecin comme un outil d'introspection.

Pour en revenir au Magicien d'Oz, le jeu est également bien présent. Tout d'abord, l'expérience scientifique prend la forme d'une modalisation de la conversation où les participants ne sont pas des usagers, mais font comme s'ils avaient besoin d'utiliser le service proposé. Néanmoins, le rôle endossé par les chercheurs situe le Magicien d'Oz dans un tout autre ordre du jeu, celui de la fabrication, c'est-à-dire, selon Erving Goffman (1991), dans un cadre d'action subi au sein duquel certains acteurs sont induits en erreur quant à ses tenants et ses aboutissants. Autrement dit, lorsque le métamessage « ceci est un jeu » est volontairement occulté pour une partie des participants. Concernant les techniques, c'est par exemple le cas du "turc mécanique ", qui peut être considéré comme l'ancêtre du PNAMBIC. Dans le domaine des expériences scientifiques, celle de Milgram, en psychologie, est également fondées sur ce principe.

En ce sens, il y a tromperie. Le Magicien d'oz, n'est pas... un magicien, ni un ventriloque, ni une créature robotisée. Les utilisateurs ne sont pas des victimes consentantes d'un procédé mimétique dont ils évaluent la qualité. Ils croient le faire. Il est bien question d'illusion plutôt que d'allusion (Grimaud 2006 :106).Ainsi, En termes de « jeu », le Magicien d'Oz exploite l'ubiquité rendue possible par l'informatique ${ }^{7}$. En revanche, il semble plus subtil qu'un simple subterfuge qui permettrait à des ingénieurs d'apprécier en amont certaines spécifications de leurs programmes. La tromperie acquiert ici une dimension heuristique en tant que simulation. En effet, elle devient un outil de mise en situation ayant vocation à extraire les comportements du participant (qui lui-même joue) du méta-contexte que représente l'expérience scientifique. Le faux permet de produire du vrai, ou plutôt il permet de produire du savoir en se rapprochant d'une réalité future, lorsque la technologie matérialisera ce qui est envisagé par la simulation humaine. Il s'agit d'une complexification pratique qui peut être analysée en termes de ritualisation.

De nombreux auteurs se sont fondés sur la théorie du jeu de Gregory Bateson pour approcher la question du rituel (voir Piette 1997). Parmi ceux-ci, Michaël Houseman et Carlo Severi, ont proposé de voir le rituel comme un mode d'action reposant sur des logiques et des mécaniques spécifiques. En ce sens, la ritualisation est une transformation par complexification du jeu à partir d'une condensation des relations (Houseman \& Severi 2009) ou des procédés d'identifications associant humains et nonhumains (Severi 2017 ; Houseman 2012 : 157-177). La ritualisation peut également tenir à une complexification du méta-message sur lequel le jeu repose (Bateson, 1977 : 211-212, Houseman 2012 : 141-153).

En reprenant l'analyse de Roberte Hamayon (2012) sur le jeu et ce qui le distingue du rituel, deux principes essentiels peuvent être associés à ces complexifications. Roberte Hamayon met en exergue une différence fondamentale entre les actions ludiques et rituelles au sens où les participants n'entretiennent pas la même relation avec le cadre de l'action lui-même, et plus précisément avec les règles constitutives (Searle, 1982) qui 
les caractérisent. Bien jouer, c'est profiter des marges du jeu, en exploiter les règles et la dimension aléatoire. C'est également ce qui valorise l'interprétation d'une œuvre par un metteur en scène ou un acteur. À l'inverse, la réussite d'une action rituelle dépend du suivi scrupuleux de normes et de prescriptions parfois minutieuses, c'est un temps de conformité (ibid. : 316). Ainsi, on joue au football, aux échecs ou au théâtre, mais on ne joue pas à un rituel ${ }^{8}$.

La seconde distinction proposée par Roberte Hamayon porte sur l'effet du jeu et du rituel. Généralement, le jeu peut évoquer ou agir sur les normes sociales, mais uniquement dans le temps et le cadre qui lui sont impartis. Le rituel à l'inverse à vocation à avoir un effet sur les personnes ou les non-humains qui y participent, sur leurs statuts ou sur leurs relations. Le jeu est avant tout une " métaphore significative " (Bateson 1977: 212), alors que le rituel est un "acte de magie sociale» (Bourdieu, 1982) $)^{9}$.

Il semble donc impropre de considérer une pratique telle que le Magicien d'Oz comme un rituel. Le Magicien d'Oz n'a pas pour conséquence de transformer les relations sociales ou les identités des participants. Le cadre de l'expérience, s'il représente lui aussi une modalisation où l'on ne joue pas, n'a pas pour dessein d'agir sur le social, mais de révéler scientifiquement la nature des choses par la maîtrise et l'analyse de ses tenants et de ses aboutissants. Cependant, cette pratique repose sur des modalités de complexification et d'identification qui peuvent être rapprochées, en termes d'action, à une forme de ritualisation relative aux identités qui y sont engagées.

\section{Le Magicien d'Oz comme personne-objet}

Supposons, à la suite de Bruno Latour (1995), qu'une des caractéristiques majeures de la pratique scientifique est l'occultation des non-humains pourtant largement impliqués dans les procédés d'expérimentation et d'innovation, autrement dit, dans la science en train de se faire. La recherche en IA serait alors avec la robotique un des rares domaines où un non-humain serait reconnu et avec lequel l'interaction serait problématisée. En ce sens, Robin Wooffitt et ses collègues (1997), mais également Nils Dahlbäck et al. (1993), insistent sur le caractère inédit de l'interaction H-M :

Jusqu'à présent, naturellement, l'analyse de l'interaction humaine a été confinée aux données de personnes parlant avec d'autres personnes. Les données sur l'interaction entre humain et machine ouvrent un nouveau champ de possibilités pour l'analyse du dialogue humain. (Wooffitt et al. $1997: 2$ )

L'adverbe "naturellement " a son importance ici. En écartant toute possibilité d'interaction humaine avec d'autres entités à l'exception de celle, récente et à venir, avec les ordinateurs, ces auteurs stipulent que celle-ci est effective, évolutive et sans précédent. Ces auteurs écartent implicitement les interactions avec les esprits ou les animaux et plus généralement toute forme de personne non humaine. Autrement dit, de leur point de vue, il y a quelque chose de révolutionnaire dans l'interaction avec la machine qui peut être rendue réelle, même lorsqu'elle est simulée par le biais du Magicien d'Oz.

Dans ce contexte pourtant, la machine est un non-humain au statut particulièrement ambigu. Premièrement, l'artefact computationnel défie la frontière entre les choses que l'on fabrique et utilise et celles grâce auxquelles on communique (Suchman $1987: 6$ ). Autrement dit, il y a une double relation à l'objet comme outil (qui médiatise l'action de 
la personne sur la matière) et comme agent (qui posséderait sa propre agency). Avec une machine, on communique tout en mesurant ses fonctionnalités. Ceci est valable pour l'informatique en général, mais une subtilité supplémentaire est à l'œuvre au sein de la recherche en IA. En effet, si l'interaction H-M est reconnue comme spécifique, comme fondamentalement différente des relations humaines, le projet scientifique qui sous-tend ce domaine de recherche a pour objectif de produire des programmes imitant le mieux possible les êtres humains. L'interaction $\mathrm{H}-\mathrm{M}$ est donc spécifique jusqu'à ce qu'elle ne le soit plus. Car, idéalement, le prototype de l'IA est une intelligence humaine, ou plus précisément un programme pouvant se rendre indistinguable d'un être humain . C'est ce qui permet la matérialisation particulière de ce non-humain dans le cadre de l'expérience du Magicien d'Oz. Non seulement les scientifiques n'occultent pas la présence du principal non-humain engagé dans leurs recherches, mais ils en endossent l'identité et font croire à son existence. Ce sont eux qui deviennent les « boites noires » de l'expérience (Latour 1995) en jouant le rôle d'un non-humain. En ce sens, la machine est là, sans être là. Par conséquent, il y a matière à voir le Magicien d'Oz, comme une personne-objet. Autrement dit, le reflet de ce que Carlo Severi nomme un objet-personne (Severi 2017).

En partant de la question de la subjectivité et de l'intentionnalité attribuée aux objets étudiées par Alfred Gell, Carlo Severi s'intéresse aux artefacts auxquels on prête la parole dans un contexte rituel et qu'il désigne comme des objet-personnes : « tout acte verbal suppose un partage d'identité : l'attribution d'états mentaux à autrui est indissociable de l'usage du langage et nous ne pouvons déchiffrer et comprendre un énoncé que si nous admettons que notre interlocuteur peut en faire autant " (Severi 2017 : 135). Se faisant, Carlo Severi propose de voir dans l'acte de parole un aspect essentiel de la personnification à partir de laquelle un artefact fait davantage que représenter une entité, en en restituant la présence . La représentation iconique se double en effet d'une désignation indiciaire (Severi 2017 : 136 ; 152) prenant la forme d'une condensation rituelle lors de laquelle il y a un jeu d'identifications complexes et de relations contradictoires.

Prenons l'exemple de Bappa, un robot permettant de projeter sa voix et son visage pour faire comme si on incarnait le dieu Ganesh (Grimaud in Grimaud et al. 2016 : 237-238), une identité complexe et ambiguë est donnée à voir par la fluctuation entre l'individu, la machine et le dieu qui la compose, et l'impossibilité de ne jamais pouvoir les différencier totalement.

51 S'il on revient au cas de la statuette funéraire antique analysé par Carlo Severi, la parole qui lui est attribuée associe une identité humaine (ou post-humaine - le défunt), sa représentation et l'identité de la personne qui, en lisant son épitaphe à haute voix, est aussi bien l'interlocuteur de la statue que celui qui lui prête la parole et constitue l'échange.

Quant est-il dans le cas du Magicien d'Oz ? Tout d'abord, la recherche en IA est fondée sur l'hypothèse qu'une équivalence de la pensée humaine peut être fabriquée, c'est-àdire qu'il est possible de concevoir une entité douée de raison avec laquelle communiquer. Cette entité n'existe pas, ou plutôt, elle n'existe pas encore. En ce sens, ce domaine de recherche a pour finalité de la faire advenir, par rapprochements successifs, grâce aux activités des chercheurs. Dans le cadre du Magicien d'Oz, cette projection dans le futur est matérialisée par le biais d'une ritualisation, où un humain 
devient le médiateur de cette absence/présence d'une machine qui non seulement devrait être là, mais surtout pourra être là.

Dans le cas de la statue funéraire, l'officiant prête sa voix à quelqu'un qui a été, en interagissant avec un artefact qui la représente. Le magicien, quant à lui, prête sa voix à un artefact qui n'est pas encore réel, en interagissant avec quelqu'un qui a été amené à croire à son existence par le truchement de sa représentation. Mais un agent conversationnel n'est pas déterminé par son apparence contrairement aux robots, il est en grande partie aniconique et incorporel. Son potentiel de présence n'est pas personnalisé par l'outil de communication qui en est le médium. Il ne relève pas de la vie ambiguë qui semble habiter le robot (Becker 2011 : 125 ; Grimaud \& Paré, 2011) et ne persiste pas hors de son usage. Eteint on lui attribue difficilement une intentionnalité. En marche, il peut exprimer ses intentions. De cette manière, les chabot survolent « la vallée de l'étrange » imaginée par Masahiro Mori (ibid.), c'est ce qui permet à un être humain de se faire passer pour l'un d'entre eux.

En ce sens, la simulation à laquelle se prête le magicien dépasse le théâtre ou la mystification, car, au delà des caractéristiques du rôle qu'il joue (une machine à écrire ou un service de renseignement), le magicien devient l'indice d'un prototype (entendu au sens de Gell 2009), c'est-à-dire la matérialisation d'une image mentale. C'est en ce sens qu'il peut être considéré comme une personne-objet. Le prototype en question est l'Intelligence Artificielle ou l'une de ces facettes, c'est-à-dire l'objectif irréalisé mais poursuivi de la fabrication d'une pensée autonome, dont l'expérience scientifique qui l'intègre est envisagée comme l'une des étapes. C'est cette indexicalité, autrement dit, ce lien entre indice et prototype de l'IA, que partagent les participants et le magicien, bien que les premiers soient amenés à croire que l'indice est réalisé par une machine, alors qu'en réalité il est joué par un être humain.

Or, cette indexicalité est socialement contrainte. Toute forme d'IA ne peut pas être simulée grâce au Magicien d'Oz. C'est là son principal paradoxe : "personne ne va croire qu'il parle à une machine s'il ne peut distinguer sa performance de celle d'un humain " (Wooffitt et al. 1997 : 33). Et pourtant c'est bien l'objectif projeté de ce domaine de recherche. Cela signifie que les formes d'IA compatibles avec l'expérience de simulation sont celles qui peuvent être reconnues comme des innovations. En effet, pour ne pas être démasqué, le magicien doit se cantonner à jouer une IA qui pourrait vraisemblablement exister au moment de l'expérience. Son rôle de machine est donc sur le fil d'une science-fiction d'anticipation pouvant passer pour une avancée scientifique et technique réelle et réalisée. Chercheurs et participants doivent ainsi partager un même prototype de l'innovation technique en IA, ce qui n'est pas toujours le cas. Cette convergence nécessaire représente également la limitation de cette technique : les caractéristiques des interactions qui y sont exécutées ne semblent pas intrinsèquement liées à l'interaction H-M elle-même, mais au degré de sophistication du langage de la machine que l'on imite. Elles dépendent de l'idée que les chercheurs se font de l'avancée technologique de simulation de l'humain par la machine qui peut être simulée par un humain sans qu'un autre ne s'en rende compte. La question des spécificités de l'interaction H-M est par conséquent relative aux indices que l'on est en mesure de produire - ou de simuler - de cette IA à un moment donné.

Or, ces indices évoluent en fonction du niveau de sophistication de la technologie depuis les années 1970 jusqu'à nos jours. Cela renvoie à l'évolution, dans les publications présentées ci-dessus, des consignes faites aux magiciens pour endosser le 
rôle de la machine. Afin que leur interprétation soit convaincante, Robin Wooffitt et al. (1997) préconisent ainsi à tout apprenti magicien de prendre un ton neutre et impersonnel et de se munir d'un programme pour rendre sa voix métallique et hachée. Or, si en leur temps, le subterfuge semble fonctionner, cela n'empêche pas les utilisateurs de considérer que cette voix de robot difficilement compréhensible est le principal défaut de conception de la machine (ibid. : 47). Vingt ans après, la voix métallique, monotone et impersonnel de Robin Wooffitt et al. (1997) a fait place aux personnalités des chatbots proposées par Indrani Mehdi Thies et al. (2017). Or, les stéréotypes que représentent ces personnalités ne font plus référence aux robots, mais à des stéréotypes humains que les participants comparent d'ailleurs à des personnages de films de Bollywood (ibid. : 452). En ce sens, ce qui est attendu de l'innovation technologique en la matière se transforme. Si le Magicien d'Oz de Robin Wooffitt et al. (1997) devait mettre de longues secondes à répondre pour simuler le temps de calcul de la machine, le chatbot simulé doit pouvoir répondre par écrit à une vitesse de conversation normale. La rupture de cadre s'est déplacée puisqu'imiter un agent artificiel perfectionné signifie imiter une machine imitant de mieux en mieux un être humain.

57 La complexification de l'action tient donc à cette identité de la machine simulée entre la présence matérielle de l'ordinateur et la projection de la possibilité d'imiter la pensée. Dans cette anticipation à l'interstice entre science-fiction et science, le magicien incarne l'image du progrès que représente l'IA en son temps, en amont des innovations technologiques.

\section{Conclusion}

Avant d'être un domaine de recherche, l'IA est un courant de pensée fondé sur l'hypothèse que la pensée peut être automatisée. Cela en fait un défi scientifique aussi bien qu'une problématique de philosophe orientés vers deux aspects essentiels : la projection et l'imitation. A partir du Magicien d'Oz, nous avons voulu mettre en lumière les logiques spécifiques qui émergent de l'articulation de ces deux aspects. En ce sens, le Magicien d'Oz tient sa pertinence heuristique, implicitement mais essentiellement, de la projection du progrès technique et scientifique. Non seulement celle au fondement de l'IA, c'est-à-dire la visée, toujours renouvelée, de se rapprocher de l'intelligence humaine, mais également l'anticipation de l'innovation technique de l'outil informatique. Il s'agit de simuler ce que l'on pense pouvoir bientôt programmer. Le Magicien d'Oz n'est pas une aberration ou un sous-produit de la recherche en IA. Il est l'aboutissement, en termes d'expérimentation, de sa logique. Ainsi, en s'affranchissant des contraintes matérielles de l'innovation technique, les chercheursmagiciens situent leur pratique dans une liminalité entre science et ritualisation, où la machine n'étant pas en mesure d'imiter son modèle, c'est au modèle de simuler ce que pourra être sa copie ${ }^{10}$. 


\section{BIBLIOGRAPHY}

Alevêque, G. et Durand, M. 2018. « Comment aborder la notion de confiance envers les objets connectés? Quelques pistes de réflexion anthropologiques », in Regards croisés sur la notion de tiers de confiance, Montpellier : Dynamique du droit : 129-148.

Amalberti, R., Carbonell, N. et Falzon P. 1993 User representations of computer systems in human-computer speech interaction. International Journal of Man- Machine Studies 38 :547-566.

Bateson, G. 1977 [1972], Vers une écologie de l'esprit, t.1, Paris : Seuil.

Baum, L. F. 1900 The Wonderful Wizard of Oz, Chicago : George H. Mill.

Becker, J. 2011 Récursions chimériques. Gradhiva 13: 113-129.

Bourdieu, P., 1982. Les rites comme actes d'institution, Actes de la recherche en sciences sociales 43 : 58-63.

Card, S.K., Moran T.P. et Newell, A. 1983 The psychology of Human-Computer interaction. Hillsdale : L. Erlbaum.

Casilli, A. 2019 En attendant les robots : enquête sur le travail du clic. Pairs : Seuil.

Chapanis, A. 1984 " Taming and civilizing computers ", Annals of the New York academy of Science, $426: 202-219$.

Corti, K., Gillespie, A. 2016 « Co-constructing intersubjectivity with artificial conversational agents: people are more likely to initiate repairs of misunderstandings with agents represented as human ». Computers in Human Behavior 58, 431-442.

Crevier D. 1997 A la recherche de l'intelligence artificielle. Paris : Flammarion.

Dafydd, G., Moore R., Winski R. 1997 Handbook of standards ressources for spoken language systems. Berlin : Mouton de Gruyter.

Dahlbäck, N., Jönsson, A. et Ahrenberg, L. 1993 Wizard of Oz studies - Why and how. Knowledgebased system 6(4) : 258-266.

Descola, P. 2005, Par delà nature et culture, Paris, Gallimard.

Dreyfus, H. L. 1972 What computer can't do : a critique of artificial reason. New York : Harper \& Row.

Flichy, P. 2003 l'innovation technique, Paris : La Découverte.

Forsythe, D. 2001, Studying those who study us. Stanford : Stanford University Press.

Fraser, N.M., Gilbert N. 1991 « Simulating speech systems ». Computer speech and language 5 : 81-99.

Gell, A. 2009 [1998] L'art et ses agents, Paris : Les presses du réel.

Goffman E., 1991 [1974] Les cadres de l'expérience, Paris : éditions de minuit.

Goffman E., 1974 Les rites d'interaction, Paris : éditions de minuit.

Gould, J.D., Conti, J. et Hovanyecz, T. 1983 « Composing Letters with a Simulated Listening Typewriter ». Communications of the ACM 26 4: 295-308.

Green, P. et Wei-Haas, L. 1985 « The Wizard of Oz : a tool for rapid development of user interfaces ». University of Michigan, rapport nUMTRI-85-27.

Grimaud, E. 2006, Têtes multiples et jeux d'optique. Terrain 46 : 85-106. 
Grimaud, E. et Paré, Z. 2011 Le jour où les robots mangeront des pommes : conversations avec un Geminoid. Paris, Éditions Petra.

Grimaud E., Taylor A-C., Vidal D., Dufrêne T., 2016, Persona. Etrangement humain, Paris, Musée du quai Branly, Actes Sud.

Hamayon R. 2012. Jouer. Étude anthropologique à partir d'exemples sibériens, Paris, Éditions La Découverte.

Houseman, M. 2012 Le rouge est le noir. Toulouse, Presses universitaires du Mirail.

Houseman, M et Severi C., 2009 Naven ou le donner à voir. Paris : MSH.

Klein, D. 1981 « Pay no attention to the man behind the curtain » Computer $14: 112$.

Klein, J., Moon, Y., Picard, R. 2002 « This computer responds to user frustration: theory, design, results, and implications » Interacting with Computers 14, 119-140.

Lassègue, J. 1998 Turing. Paris : Les belles Lettres.

Latour, B. 1995 [1987] La science en action. Paris : la Découverte.

Luger, E. et Sellen, A. 2016 "Like Having a Really bad PA": the gulf between user expectation and experience of conversational agents. In, Proceedings of CHI 2016: 5286-5297.

Malhotra, A. et Sheridan, P. 1976 « Experimental determination of design requirements for a program explanation system ». IBM rapport $n^{\circ} 5831$.

Marquis, P. Papini, O. et Prade H. 2014, Panorama de l'intelligence artificielle, ses bases méthodologiques, ses développements, vol. 1. Toulouse Cepadues.

Medhi Thies, I., Menon, N., Magapu S., Subramony M., et O’Neill J. 2017 « How Do You Want Your Chatbot? An Exploratory Wizard-of-Oz Study with Young, Urban Indians ». INTERACT 2017 : 441-459.

Neff, G. et Nagy P. 2016 « Talking to Bots: Symbiotic Agency and the Case of Tay », International Journal of Communication 10, 4915-4931.

Newell A. F., Arnott L., Carter K., Cruickshank G., 1990, Listening typewriter simulation studies, International Journal of Man-Machine Studies $\mathrm{n}^{\circ} 33$ pp. 1-19.

Piette A., 1997, Pour une anthropologie comparée des rituels contemporains, Terrain $\mathrm{n}^{\circ} 29 \mathrm{pp}$. 139-150.

Reeves, B., Nass, C. 1996 The Media Equation: How People Treat Computers, Television, and New Media Like Real People and Places. New York : Cambridge University Press.

Russell S. et Norvig P. 2010 Intelligence artificielle. Paris : Pearson.

Sacks, Harvey 1992 Lecture on Conversation. Oxford : B. Blackwell.

Salembier, P. 1991 « Le Magicien d'Oz une technique de simulation et

d'investigation des situations d'interaction avec un système « intelligent » ", in Y. Schegloff, Emanuel 1968 Sequencing in conversational openings. American anthropologist 70 :1075-1095.

Searle, J.R. 1980 Minds, Brains and programs : The Behavioral and Brain Sciences,

vol. 3, Cambridge University Press, 1980.

Searle, J.R. 1982 Sens et expression. Paris, les Editions de Minuit.

Severi, C. 2017 L'objet-personne. Paris : Éditions rue d'Ulm. 
Small, D.W. et Weldon, L.J. 1983 « An experimental comparison of natural and structured query languages ». Human factors 25(3) : 253-263.

Suchman, L.A. 1987. Plans and situated actions: the problem of human-machine communication Cambridge : Cambridge University Press.

Wallace R.S. 2008 « The anatomy of ALICE », in Epstein, R., Roberts, G., Beber, G. Parsing the turing test. NewYork : Springer : 181-210.

Weizenbaum, J. 1966 « ELIZA - A Computer Program for the Study of Natural Language Communication between Man and Machine » Communications of the Association for Computing Machinery $9: 36-45$.

Wooffitt R., Fraser, N.M., Gilbert N. et McGlashan S. et 1997 Human, computers and wizards. Analysing human (simulated) computer interaction Londres : Routledge.

Zoltan-Ford, E. 1984 « Reducing Variability in Natural-Language Interactions with Computers », Proceedings of the Human factor society : 768-772.

\section{NOTES}

1. En comparaison avec les phénomènes plus généraux analysés par Patrice Flichy (2003: 179-233).

2. Au sens d'Alfred Gell (2009).

3. Du point de vue de la machine, cette question des spécificités de l'interaction a été posée par plusieurs auteurs, en particulier John Searle (1982) qui soulève le problème des actes indirects de parole. Il s'interroge ainsi sur la possibilité pour les machines d'identifier ce qui relève d'une requête étant donnée certaines formes de politesse en langage naturel. En ce sens, d'un point de vue computationnel, la réponse logique à la question « pouvez-vous me passer le sel ? » n'est pas un acte mais « oui » ou « non ».

4. https://news.microsoft.com/apac/features/much-more-than-a-chatbot-chinas-xiaoice-mixesai-with-emotions-and-wins-over-millions-of-fans/ consulté le 29 janvier 2019.

5. Avant d'être déconnecté au bout de seulement 24h, Tay avait envoyé plus de 90000 messages.

6. On parle de « méta-communication » pour « tout message qui, implicitement ou explicitement, définit un cadre, [et] fournit ipso facto, au receveur, instructions et appuis dans sa tentative pour comprendre les messages qui y sont contenus (Bateson, 1977: 214). L'ironie par exemple repose sur ce type de «méta-messages ».

7. En ce sens, le Magicien d'Oz anticipe le piège à affordance qui caractérise les objets connectés, car leur fonctionnement repose sur un réseau de tiers incertains, dont le nombre et la qualité sont rendus indiscernables par l'ubiquité numérique (Alevêque et Durand, 2018).

8. Notons ici que les programmes d'intelligence artificielle donnent l'impression de jouer à des jeux, alors qu'ils ne font qu'en suivre scrupuleusement les règles. Pourtant, Joffrey Becker montre que lors des jeux impliquant les robots ce qui est apprécié par les spectateurs est également la faculté des ingénieurs à déléguer aux machines leur propre intelligence du jeu (2011).

9. Un rite peut néanmoins intégrer dans son cadre des éléments de théâtralisation, comme dans le cas du Naven Iatmul (Houseman et Severi 2009). Et un jeu peut avoir "valeur de rite» (Hamayon, 2012), ce qui explique pourquoi ils soient si ardus à distinguer. 
10. Je remercie le Human at Home Project (Université de Montpellier et Université Paul Valéry) qui a financé cette recherche postdoctorale.

\section{ABSTRACTS}

Artificial Intelligence research is based on specificities that the major debates that have accompanied its development since the 1950s have expressed without, however, allowing them to be precisely defined. By focusing on the scientific practices themselves and in particular on a form of experience that is specific to this field, this article shows how the tension represented by bringing the machine closer to the human being can produce forms of ritualization within science. The Wizard of Oz technique, which appeared in the 1970s, is based on the interaction of a human with a simulated machine in order to anticipate computer progress and its consequences. By playing the role of the machine, the researcher materializes an unrealized innovation that refers to a non-human - the AI - whose future existence is projected.

La recherche en Intelligence Artificielle repose sur des spécificités, que les grands débats qui accompagnent son développement depuis les années 1950 expriment sans toutefois permettre de les circonscrire avec précision. En s'intéressant aux pratiques scientifiques elles-mêmes et en particulier à une forme d'expérience qui est propre à ce champ d'études, cet article montre comment la tension que représente le rapprochement de la machine à l'être humain peut produire des formes de ritualisation au sein de la science. Apparue dans les années 1970, la technique du Magicien d'Oz est en effet fondée sur l'interaction d'un humain avec une machine simulée dans le but d'anticiper les progrès de l'informatique et leurs conséquences. En jouant le rôle du programme, le chercheur matérialise une innovation irréalisée qui renvoie à un nonhumain - l'IA - dont on projette l'existence future.

\section{INDEX}

Mots-clés: Intelligence artificielle, Expérience, Ritualisation, Interaction humain-machine, Simulation

Keywords: Artificial Intelligence, Experimentation, Ritualization, Human-Computer Interaction, Simulation

\section{AUTHOR}

\section{GUILLAUME ALEVÊQUE}

Guillaume Alevêque est anthropologue associé à l'Institut interdisciplinaire d'anthropologie du contemporain (IIAC) de l'Ecole des hautes études en sciences sociales. Ses recherches portent sur la production de la connaissance et les pratiques collectives et usages politiques qui lui sont associés à partir de deux sujets distincts, d'une part le militantisme écologiste et culturel en Polynésie française face à la question du colonialisme et du nucléaire, et d'autre part l'impact des 
innovations technologiques sur la recherche scientifique en Intelligence artificielle (IA) et Internet de objets (IoT). 